\title{
Summary of Primary Culture of Human Lung Cancer Cells
}

Dai Y', Guo S', Huang S', Zhang J ${ }^{2}$ and Zhang $Z^{2 *}$ ${ }^{1}$ Second Clinical College, Guangzhou Medical University, China

${ }^{2}$ Radiology Department, The Second Affiliated Hospital of Guangzhou Medical University, China

*Corresponding author: Zhenfeng Zhang, Radiology Department, Guangzhou Medical University, Haizhuqu District, Guangdong, China

Received: October 25, 2018; Accepted: November 28, 2018; Published: December 05, 2018

\begin{abstract}
Lung cancer is one of malignant tumors in the world with the highest morbidity and mortality [1]. Individualized treatment of lung cancer depends on the establishment of primary lung cancer cell lines. Primary cell culture of lung cancer has been studied for more than 30 years, but the success rate is still very low. This article reviews the research on primary culture of lung cancer cells both at home and abroad. The methods of isolation, purification, biological characterization and culture of lung cancer cells were discussed respectively. By comparing the advantages and disadvantages of different primary culture schemes, we hope to get the Enlightenment of better scheme and provide reference value for clinical and cell research.
\end{abstract}

Keywords: Lung cancer; Methods of primary culture

\section{Introduction}

\section{The isolation of lung cancer cells}

The use of isolation methods for lung cancer cells will roughly determine the rate of obtaining lung cancer cells, which will be helpful to the purification of lung cancer cells at later stage and the purpose test of relevant cell lines. With regard to the isolation of lung cancer cells, taking into account the simplicity associated with the experiment and the cost of the experiment, the basic method has been continuously adopted by the vast number of experimenters and continue to improve from the more traditional method of direct separation to the collagenase perfusion method created by Seglen. In the following passage, some reasonable improvement experiments will be explained on the basis of the general method.

Non-enzymatic digestion: Direct separation: The direct separation method refers to the general mechanical method to separate the cells in the animal tissue mass and to separate the target cells from the target cells by the appropriate mechanical method. Shearing, oscillating and pressing are often used to disperse the tissue and cells, and centrifugation is used to separate the cells. In the experiment of lung cancer cell separation, the direct separation method will face the defect of low acquisition rate (the direct death of the target cell in mechanical separation), but the cost of the direct separation method is lower and the operation is simpler.

The tissue mass culture: Tissue block culture is one of the methods of cell culture, but it can also be used as an effective method for cell separation because of the migration of non-target cells from the tissue mass in the process of tissue mass culture [2]. According to some operational differences, it mainly includes reverse drying and thin layer culture.

Reverse drying: The obtained tissue was cut into tissue blocks about the size of $1 \mathrm{~mm}^{3}$, washed with PBS buffer for 3 times and transferred to the culture bottle at a certain distance. The culture bottle was flipped gently to prevent the tissue mass from sliding, and the culture medium was added to the non-cell growth surface of the culture bottle. Plug the bottle and place it in a $37^{\circ} \mathrm{C}$ incubator for $2-3$ hours to dry up the small pieces. After taking out the bottle, open the bottle and hold the culture bottle 46 degrees diagonally, gently inject a small amount of culture fluid into the bottom foot of the bottle, then slowly turn the culture bottle over and start the culture. With the increase of cell value, the medium can be added appropriately.

Thin layer culture: The obtained tissue was cut into tissue blocks about the size of $1 \mathrm{~mm}^{3}$ and washed with PBS buffer for 3 times and transferred to the culture bottle at a certain distance. After 30 minutes, fresh $2.0 \mathrm{~mL}$ medium was injected slowly. Culture the tissue in $37^{\circ} \mathrm{C}$ incubator without floating, and replace the new medium every 2-3 days.

Enzyme digestion: Collagenase digestion: Luo Mingzhi systematically divided collagenase conversion methods into three categories: two-step in situ collagenase perfusion method [3], direct collagenase digestion method and semi-in-situ perfusion method. At the same time, the advantages of Polyvinylpyrrolidone (PVP) in the separation of cell mass formed by single cell aggregation are also described. However, in the separation experiment of lung cancer cells, because of the different materials, the corresponding collagenase conversion method is also different. The following is the general method for the isolation of lung cancer cells from lung cancer tissue.

Lung cancer tissue obtained from biopsy is an important source for lung cancer related experiments. About $1 \mathrm{~cm}^{3}$ of lung cancer tissue without necrosis was taken and immediately put into frozen 1640 medium with appropriate concentration of penicillin $G$ and streptomycin to ensure the activity of tissue mass cells. After being transported to the laboratory and removed blood clots, the blood clots were washed with PBS buffer for 2 times, cut into tissue blocks of $1 \mathrm{~mm}^{3}$ size, digested with collagenase, bathed in water at $37^{\circ} \mathrm{C}$ for one hour, passed through $38 \mu \mathrm{m}$ sieve to obtain cell suspensions, washed twice, and centrifuged. The cells obtained from the supernatant are diluted into cell suspensions. Perco could also be used for further purification. 
Collagenase digestion could simulate the cellular environment. The damage of tissue treated by collagenase is limited and reversible in the process of digesting the stroma of the cells, compared with the conventional mechanical treatment.

The survival rate of the cells treated with this method is greatly improved, and thus the rate of lung cancer cell acquisition will also be increased.

The use of other enzymes: Joseph Seo [4] raised the problem of using enzymes to isolate lung cancer cells. They used many enzymes, such as collagenase, disperse enzyme, trypsin, and so on, but the results were not satisfactory, because it was difficult to get a single cell population after enzyme treatment. In their conclusion, they pointed out two shortcomings:

- The separation of simple cancer cells from fibroblasts was treated only by dissipating enzymes, but a large number of fibroblasts still remained on the disk.

- Trypsin-EDTA simultaneously isolated peripheral fibroblasts with a long time.

\section{Purification methods and biological characters detection of primary lung cancer cells}

Purification method of primary lung cancer cells: No matter which method is used to isolate lung cancer cells, there is a problem of lung cancer cell purification, mainly to remove fibroblasts [5]. In primary culture, the elimination of fibroblasts is one of the keys to the success of lung cancer cell culture.

A study indicates [4] that media containing serum or growth factors promote the growth of fibroblasts, making it difficult to purify the required cell population in the co-culture state. H. BaillieJohnson et al. [6] cultured Small Cell Lung Cancer (SCLC) cell lines and found that when these fibroblasts began to overgrow, to transfer specimens growing in HITES+2.5\% FCS to separate HITES alone for some period of time could inhibit fibroblast growth. PingWang et al. [7] found that the culture medium of Defined Keratinocyte-Serum Free Medium (DK-SFM) did support the selective growth of typical epithelial cells and significantly inhibited the growth of fibroblasts coexisting. Joseph Seo et al. [4] indicated that replacing serum with N2 supplements prevented fibroblast proliferation while allowing lung cancer cells to grow. As a result, the N2-supplemented was superior in purification while the BEI medium of $10 \%$ FBS was the first-rank culture medium in proliferation.

Most studies are based on the method of trypsin enzymatic digestion and differential attachment to isolate and purify fibroblasts by, so as to purify cancer cells [8]. For example, according to the characteristics that tumor cells are slower than fibroblasts to adhere to the wall, the cell suspensions containing the two types of cells are repeatedly adhered to the wall of dish with nutrient solution without serum, so that the two types of cells separated from each other. The general method of operation is similar to that of cell passage. 1) When the cells have grown to a certain amount, the medium is inhaled. Then digest with $0.25 \%$ trypsin/EDTA and observe with microscope. When the fibrocytes have been shed, the digestion is terminated by adding one times the volume of trypsin to the medium. Epithelioid cells continue to adhere to the medium. Wash twice with PBS, add serum- free medium and blow to make cell suspension; 2) Fetch three tissue culture flasks, named separately from A to C; first, the suspension is inoculated in A culture bottle. After half an hour of static culture in the incubator chamber, gently tilt the culture bottle, let the liquid concentrate in the bottle corner, slowly suck out all the culture liquid, and then inoculate it into the B culture bottle. Add a little of the complete culture solution to bottle A and maintain the culture in the incubator chamber. 3) After half an hour of cell culture in B bottle, the culture solution is poured into bottle $\mathrm{C}$ in the way of treating bottle A. Then add complete culture solution to bottle B. When all three bottles contain the medium, the culture s continued in the incubator. If the operation is successful, the next day's observation shows that bottle A is mainly fibroblasts bottle B is mixed with two types of cells, and bottle $\mathrm{C}$ is probably mainly cancer cells. If necessary, it can be repeatedly treated until the cancer cell purification.

In addition, some studies use anti-fibroblast columns to isolate fibroblasts [9].

On the other hand, the study by HirokoEndo et al. [10] also demonstrated that using the CTOS (Cancer Tissue-Originated Spheroid) method could obtain primary lung tumor cells of high viability and purity compared with conventional culture methods. CTOS could be a new platform for the study of lung cancer biology. However, it is still necessary to optimize the tissue separation protocol to improve the preparation of CTOS from tiny tissue samples of lung cancer.

\section{Biological characters detection of primary lung cancer cells}

Identification of lung cancer cells: Due to the heterogeneity and variability of tumor cells [11], every cancer patient needs to be seen as an individual with a unique disease. In order to achieve an effective individualized strategy in clinical practice, it is important to elucidate the biological behavior characteristics of individual lung cancer cells. In general, the characteristics of lung cancer cells are analyzed and identified to evaluate whether the cells had the main characteristics of tumor cells by means of routine light microscopy, H\&E (hematoxylineosin) staining, immunocytochemistry, immunohistochemistry, nude mice xenograft model and soft agarose assay $[8,12]$. After repeated cell passage, the characteristics of primary lung cancer cells can be altered. Therefore, it is best to describe the biological characteristics of lung cancer cells in early generations.

David P. Kodack et al. [9] developed an immunofluorescence analysis method to identify NSCLC cells in mixed culture using a mixture of cytokeratin 8 and cytokeratin 18 monoclonal antibodies. In addition, they found that exogenous EGF and insulin could reduce the sensitivity of EGFR mutant and ALK-translocated NSCLC cells to EGFR or ALK inhibitors respectively. Therefore, it was essential to remove them from the culture medium before drug sensitivity analysis.

Zengli Zhang et al. [13] established long-term cultivation conditions of Patient-Derived Tumor Spheroid (PDS), which allowed the cytological features and markers of primary tumors to be recapitulated. The PDS established by 3D culture were shown by H\&E staining to be tumor cells with high nuclear-cytoplasmic ratios, hyper chromatic nucle and irregular nuclear membrane. Immunostaining 


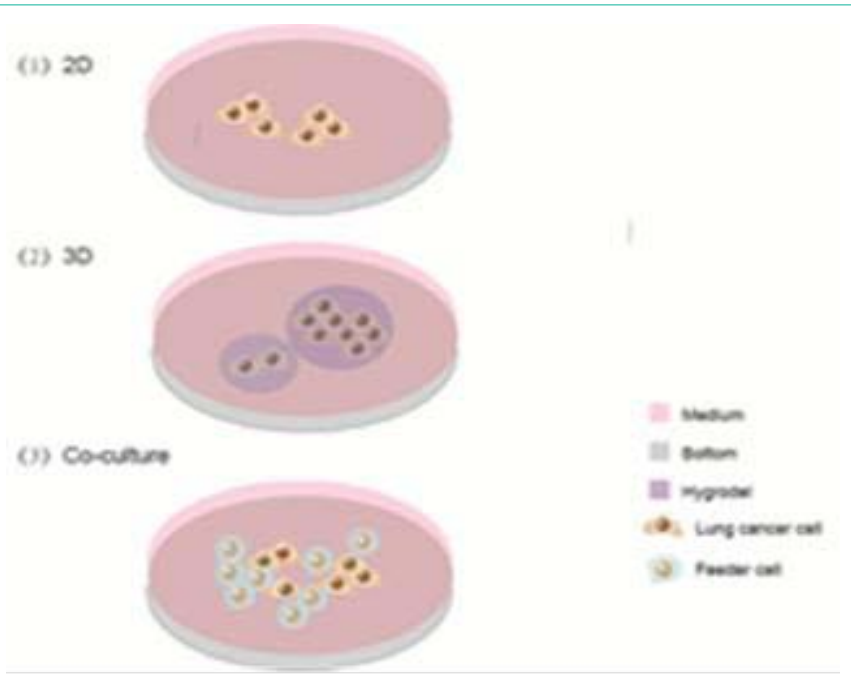

Figure 1:

showed that their PDS cultures all stained positive for lung tumor marker TTF-1, confirming the origin of adenocarcinoma of the tumor. HE and immunohistochemical staining of multigenerational PDS in their culture also showed that the cytological characteristics of tumor cells remained stable and the clinical markers were consistently retained with serial passaging. In the future, they will conduct further studies on the comprehensive characterization of gene expression profiles and mutations in tumor spheres derived from patients to prove that the model can also generalize the molecular and genetic diversity of NSCLC tumors. The Asian patient-specific NSCLC study is also expected to be carried out.

Identification of lung cancer cytogenetics: It is reported [10] that long-term culture of lung cancer cells in vitro will lead to accumulation of genetic variation. To confirm that the cultured tumor cells are derived from parental tumors, and to assess the genetic variation between the patient's tumor and the established model, which lays a foundation for screening targeted drugs and accurate treatment, experiments such as chromosome analysis, FISH (Fluorescence In Situ Hybridization), DNA fingerprinting and genomic DNA sequencing can be conducted. WES (Whole-Exome Sequencing) is an important approach to understand tumor diversity [12]. Yanan Jiang et al. [14] found in the whole-exon sequencing results that primary cell lines and the PDX (Patient-Derived Xenografts) model established with LG50 retained the genetic characteristics of more than $90 \%$ of patient tumor tissues, while, pointing out that more samples are needed to verify that result.

On the one hand, recent studies have shown that, CAFs (Carcinoma-Associated Fibroblasts) have intra-tumoral or interindividual heterogeneity and promote tumor progression in a number of ways, having attracted more and more attention as new targets for cancer treatment. The study by Masafumi Horie et al. [10] showed that it was necessary to further characterize heterogeneous CAFs from cancer patients, as the prognosis of patients could be predicted due to tumor stromal 1 features, such as gene signature.

Joseph Seo et al. [4] pointed out tumor staging affects the expression of EMT (Epithelial-Mesenchymal Transition) markers.
EMT was important in tumors because it promoted metastasis. Therefore, it was equally important to study its biological changes during and after EMT.

Even in the same patient genetically defined, tumors also show heterogeneity in response to targeted therapy [9]. In vitro drugsensitivity test of tumor cells is an important basis for individualized treatment of patients, by which cytotoxicity of anticancer drugs is detected and the most effective chemotherapy drugs or combinations of chemotherapy drug are selected. Studies demonstrate a good concelation between that drug sensitivity test in vitro and clinical sensitivity.

Using MTT biological characteristics, Msomann established the MTT method for cell activity test, which was then introduced into the sensitivity test of anticancer drugs for tumor cells [15]. MTT method is characterized by high sensitivity, good repeatability, economy, high speed, economy, automation, non-radiation pollution, simple operation and reduced experimental error compared with other in vitro drug sensitive test methods, such as cytometry, soft agarose assay and test incorporated with $3 \mathrm{H}-\mathrm{TdR}$, etc. In addition, it has a good consistency with clinical chemotherapy efficacy and is conducive to clinical application [16]. For this reason, Fraser L [17] proposed to use culture plate coated with cell matrix to make cells grow with adherence to reduce the error caused by cell loss in MTT test. In addition, the primary cultured cell population is a mixture, and the effective purification can make the result of the method more accurate. However, David P. Kodack et al. [9] found that drug sensitivity in mixed celcultures could be qualified by an immunofluorescencebased assay. They found that the ability to detect drug sensitivity in a culture medium containing fibroblasts and tumor stromal cells overcomed the time problem of using a culture medium without matrix. In addition to accelerating the time from biopsy to drug testing, this ability, due to the presence of tumor stromal cells, also allowed for potentially better tumor response performance, and increased the success of the sample, although it also presented greater challenges for the establishment of a pure cancer cell line.

On the other hand, Zengli Zhang et al. [13] pointed out that compared with the 2D (two-dimensional model), the 3D PDS model could better reflect the real interaction between drugs and tumor tissues. When they studied the cytotoxicity of cisplatin in $3 \mathrm{D}$ cultures and $2 \mathrm{D}$ cultures using Cell Turter GLO 3D assay, they found that the IC50 value is much higher than that reported in most $2 \mathrm{D}$ non-small Cell lung cancer Cell lines. In addition, in the fields of pharmacology, cell biology and cytotoxicity, electric potential and current can be applied to the cell-based system to monitor biological activity, which demonstrates the feasibility of applying new electrochemical biosensors to the study of three-dimensional cell responses of drug compounds. Hoon Jeong et al. [17] showed that biosensor monitoring based on three-dimensional cells could be used as an alternative method for screening high-throughput drug discovery.

Although the use of 3D systems avoids over-or under-estimation of specific drugs in terms of drug sensitivity and resistance, as well as drug dosages, the efficiency, longevity, repeatability and comfort of work in many $3 \mathrm{D}$ methods are worse than $2 \mathrm{D}$ systems. Vinci et al. [18] solved the problem of low reproducibility in three-dimensional culture by describing a functional assay method based on three- 
dimensional sphere for tumor target validation and drug evaluation. They used 96 holes of ultra-low attachment plates, each of which produced only one spheroid. The size of the obtained spheroids was reproducible and showed Gaussian distribution

Most studies on the mechanism of drug resistance are carried out on drug-resistant tumor cell lines. However, the growth pattern and biological characteristics of long-handed drug-resistant cell lines have changed to a certain extent, which makes the cell's sensitivity to drugs not completely consistent with the tumor's response to chemotherapy in vivo, thus losing the advantage of drug sensitivity test guiding clinical chemotherapy. Since the introduction of serumfree chemically defined media, many researches have explored the establishment of efficient and stable long-term culture system of human lung cancer cells in vitro [12]. Future drug susceptibility testing and resistance mechanism studies will be based on a larger number of clinical samples in order to further improve the effectiveness of individualized treatment for patients with advanced lung cancer.

\section{Cultural Program of Lung Cancer Cell}

The primary culture methods of human lung cancer in the past 30 years can be divided into 2-D culture model, 3-D culture model and cell co-culture system. The most commonly used is 2-D culture model, but there are also some limitations. Therefore, 3-D culture model and cell co-culture system are becoming more and more popular.

\section{2-D culture model}

Attached to the two-dimensional culture medium, cells grow monolayer in Petri dishes or flat Petri dishes and adhere to the surface of plastic. Most studies on cancer biology are based on the use of $2 \mathrm{D}$ cell culture in vitro.

At home and abroad, the most common steps of $2 \mathrm{D}$ culture model are: Fresh lung cancer tissues were obtained by surgery and washed after mechanical isolation. They were cultured directly in medium or enzymatic hydrolysis into single cells and then cultured in medium. The details of digestive enzymes, digestive time, and medium composition vary from laboratory to laboratory, and the results vary accordingly. For example, the lung cancer cell 2D culture model introduced by Ping Wang et al. [7] is: Fresh lung cancer tissue resected surgically was washed repeatedly with RPMI- 1640 containing penicillin and streptomycin. Necrotic and blood vessel tissue was eliminated. The tissue was cut into about $1 \mathrm{~mm}^{3}$ with sterile scissors and placed in a $25 \mathrm{~cm}^{2}$ culture flask. RPMI- 1640 medium containing $200 \mathrm{U} / \mathrm{mL}$ collagenase type II was used to digest collagenase for 2-4 hours in a $5 \% \mathrm{CO}_{2}$ and $37^{\circ} \mathrm{C}$ incubator. When most of the cells were digested into a single cell, they were washed with RPMI-1640 and centrifuged three times under $300 \mathrm{~g}$ centrifugal forces to completely remove collagenase. Lung cancer cells were suspended in Defined Keratinocyte-Serum Free Medium (DK-SFM) with Glutamine, EGF $20 \mathrm{mg} / \mathrm{ml}$, basic-FGF $10 \mathrm{mg} / \mathrm{ml}, 2 \%$ B27, PS and amphotericin B $0.25 \mathrm{mg} / \mathrm{ml}$ and transferred to a $25 \mathrm{~cm}^{2}$ flask. The cells were cultured in a $5 \% \mathrm{CO}_{2}$ and $37^{\circ} \mathrm{C}$ humidifying incubator. Fresh medium was replaced every 2-3 days. When the cell density was $80 \%$, passaged after digesting by TrypLEEM Express. Primary cell lines maintained under these conditions had the longest passage time, exceeding 30 generations, and showed no signs of growth decline.

In addition, there is also biopsy with bronchofibroscopy in bronchial cavity for primary culture of lung cancer cells, or puncture to obtain pleural effusion culture, etc. The procedure of specimen preparation and culture is similar to that of surgical tissue block. However, the number of lung cancer cells obtained by bronchoscopic biopsy and pleural effusion is small, and the results of culture are mostly disappointing.

\section{3-D culture model}

Three-dimensional culture, in which cells from donor tissues are cultured in three-dimensional structures of cells, mimics the structure of parental tissues more accurately than two-dimensional models. Over the past five years, many advances have been made in 3D-cultured patient-derived tumor models, most of which have been modified to grow benign intestinal organs from the initial use of LGR5 + intestinal stem cells. According to the method of preparation, the 3D model can be divided into: suspension culture on non-adherent plates, culture in concentrated medium or gel material, and culture on scaffolds.

Spheroid formation (suspension culture or soft agar culture) is a common method to evaluate the role of genes in the self-renewal and maintenance of tumor stem cells. For example, Cancer TissueOriginated Spheroid (CTOS), introducted by Hiroko Endo, is: By surgical resected, lung cancer tissue was mechanically separated into about $1 \mathrm{~mm} 3$ and cleaned with Hank 's balanced salt solution. The specimens were transferred to $100 \mathrm{ml}$ glass flask and digested in DMEM medium with $0.26 \mathrm{U} / \mathrm{ml}$ of releasing enzyme $\mathrm{DH}$ and $1 \%$ penicillin-streptomycin for 1-2 hours. Digested tissue suspensions were filtered through 500 and 250 micron mesh filters to remove large amounts of undigested debris and then further through 100 and 40 micron cell filters. Digested tissue suspensions were filtered through 500 and 250 micron mesh filters to remove large amounts of undigested debris and then further through 100 and 40 micron cell filters. The debris on the cell filter and the cells in the mobile component were collected and washed with Hank's equilibrium salt solution respectively, and cultured in StemPro hESC medium. The culture success rate of the system was high, 100 of 125 cases were successful, and the characteristics of the original tumor could be retained. At the same time, the CTOS method is also suitable for the culture of pleural effusion. The success rate is considerable, and 8 of 18 cases are successful.

In addition, Zengli Zhang et al. [13] established long-term culture conditions for Patient Derived Tumor Spheres (PDS) from patients with NSCLC, allowing a re-generalization of the cytological characteristics and markers of primary tumors, and demonstrating the usefulness of drug screening for PDS. The resected lung cancer tissues were isolated and digested in $5 \mathrm{mg} / \mathrm{ml}$ collagenase $\mathrm{I}$ at $37^{\circ} \mathrm{C}$. The cells were plated in 96-well plate with a density of $3 \times 10^{3} \mathrm{cell} /$ pore and stored in humidifying incubator at $5 \% \mathrm{CO}_{2}$ and $37^{\circ} \mathrm{C}$. The medium was supplemented with $1 \% \mathrm{FBS}, 10 \% \mathrm{~N}-2,200 \mathrm{mg} / \mathrm{ml}$ of Naoggin, B27, N-acetylcysteine, $100 \mathrm{U} / \mathrm{ml}$ of penicillin and $100 \mathrm{ug} /$ $\mathrm{ml}$ of streptomycin DMEM/F12. And the PDS cultures can be easily amplified and frozen.

\section{Establishment of co-culture}

Methods of cell co-culture can be divided into direct and indirect. Direct co-culture mimics the tumor microenvironment, and different cell types grow together in the same environment. Indirect co-culture 
is the separation of cells from physical barriers. These two can be carried out in 2D system or 3D system. Compared with other primary culture methods, co-culture is easy to operate, having high success rate, less money, less cell demand, rich source of samples, short culture cycle, and widely used in the field of cancer.

Conditionally Reprogrammed Cells (CRCS) was recommended by Boning Gao et al. [19] Lung cancer cells isolated from lung cancer tissues of patients and 3T3 fibroblasts irradiated by X-ray were cultured together in a pre-packed murine collagen flask. And the conditioned medium was a 3:1 F12/Dulbecco's modified Eagle's medium containing $5 \%$ fetal bovine serum, $0.4 \mu \mathrm{g} / \mathrm{mL}$ hydrocortisone, $5 \mu \mathrm{g} / \mathrm{mL}$ insulin, $8.4 \mathrm{ng} / \mathrm{mL}$ cholera toxin, $10 \mathrm{ng} / \mathrm{mL}$ epidermal growth factor, and $24 \mu \mathrm{g} / \mathrm{mL}$ adenine with addition of 5 to $10 \mu \mathrm{mol} / \mathrm{L}$ Y-27632. Because 3T3 fibroblasts are sensitive to trypsin, we can use different enzymolysis time of trypsin to isolate lung cancer cells and $3 \mathrm{~T} 3$ fibroblasts, that is, $0.05 \%$ trypsin to digest for 30 seconds to one minute, tap the bottom of the culture bottle can make $3 \mathrm{~T} 3$ fibroblasts shed. But the cancer cells were still adherent. Every 4-6 days to replace the new 3 T3 fibroblasts. The success rate of this culture system is $88 \%$. Of the 29 cases, 18 were successfully cultured with lung cancer cells and 3T3 fibroblasts by Jill M. Siegfried [20], and the success rate was high.

Defined by David P. Kodack [9], "feeder+TCM" is by co-culture of tumor with tumor stromal cells and fibroblasts, the functional test was successfully completed within one month after the collection of samples from Non-Small Cell Lung Cancer (NSCLC). The culture medium they defined is TCM medium coated with irradiated human foreskin fibroblasts. Fibroblasts are as trophoblast cells. TCM medium contains F/12375 mL, L-glutamine $125 \mathrm{~mL}$, glucose $4.5 \mathrm{~g} / \mathrm{L}$, sodium pyruvate-free $4.5 \mathrm{~g} / \mathrm{L}, 5 \% \mathrm{mg} / \mathrm{L}, 1 \% \mathrm{mg} / \mathrm{mL}$ hydrocortisone, $4 \mathrm{mg} / \mathrm{mL}$ hydrocortisone, $1 \mathrm{mg} / \mathrm{mL}$ insulin, $10 \mathrm{ng} / \mathrm{mL}$ epidermal growth factor, $1.68 \mathrm{ng} / \mathrm{mL}$ cholera toxin, $12 \mathrm{mg} / \mathrm{L}$ adenine, $10 \mathrm{mg} / \mathrm{mL} \mathrm{Y-27632.}$ They found that feeder + TCM media tended to be superior to other media in the process of tumor cell line formation, such as RPMI media containing $10 \%$ FBS coated with collagen (R10), DMEM media containing $10 \%$ FBS coated with collagen (D10), A CL4 medium containing $<5 \%$ FBS coated with collagen $(\mathrm{A}<5)$, and RPMI medium coated with collagen (R10). The ACL4 medium containing more than $5 \%$ FBS $(A>5)$. Feeder+TCM's training program brings the gospel to cancer cell sensitivity assessment on the other hand.

Dr. Michael Kapinsky said, when inactivated 3T3 fibroblasts were co-cultured with lung cancer cells, Conditioned Medium (CM) derived from the establishment of lung cancer cell line A549 was used as a supplement to the culture medium of primary solid lung cancer cells. CM was found to have Transforming Growth Factor (TGFa) and Insulin-like Growth Factor-I (IGF-I) can stimulate the formation and growth of monolayer colonies in semisolid medium of cultured protoplast tumor cells.

\section{Conclusion}

Lung cancer cell lines are important tools for elucidate the pathogenesis of lung cancer and develop new therapies [21]. At present, there are various forms of cell culture, so it is possible to choose suitable methods for research purposes. The most common research mode is two-dimensional culture system. However, due to its limitations, 2D culture is increasingly considered to be an inefficient model for studying this process - related to cell exposure to ionizing radiation or chemotherapy drugs [10]. Multicellular and 3D models may be a better way to look for new biomarkers and new therapeutic strategies, bringing us closer to the goal of personalized medicine. Ultimately, the failure of cell culture was as follows: no cancer cells were found after 6 months of culture; as opposed to the success of culture, cancer cells no longer required fibroblast donor layer growth, no matrix fibroblasts (visible to the naked eye), could be frozen and thawed and then stabilized and kept the same drive as the original biopsy specimen [9].

\section{References}

1. Siegel R, Naishadham D, Jemal A. Cancer statistics for Hispanics/Latinos, 2012. CA: A Cancer Journal for Clinicians. 2012; 62: 283-298.

2. Matsuura H. Primary cultured cells and an established cell line of human hepatocellular carcinomas. Acta medica Okayama. 1983; 37: 341-352.

3. Reese JA, Byard JL. Isolation and culture of adult hepatocytes from liver biopsies. In vitro. 1981; 17: 935-940.

4. Seo J, Park SJ, Kim J, Choi SJ, Moon SH, Chung HM. Effective method for the isolation and proliferation of primary lung cancer cells from patient lung tissues. Biotechnology letters. 2013; 35: 1165-1174.

5. Gazdar AF, Oie HK. Cell culture methods for human lung cancer. Cancer genetics and cytogenetics. 1986; 19: 5-10.

6. Baillie-Johnson $\mathrm{H}$, Twentyman PR, Fox NE, Walls GA, Workman P, Watson $\mathrm{JV}$, et al. Establishment and characterisation of cell lines from patients with lung cancer (predominantly small cell carcinoma). British Journal of Cancer. 1985; 52: 495-504.

7. Wang $P$, Gao Q, Suo Z, Munthe E, Solberg S, Ma L, et al. Identification and characterization of cells with cancer stem cell properties in human primary lung cancer cell lines. PloS one. 2013; 8: e57020.

8. Si LL, Lv L, Zhou WH, Hu WD. Establishment and identification of human primary lung cancer cell culture in vitro. Int J Clin Exp Pathol. 2015; 8: 65406546.

9. Kodack DP, Farago AF, Dastur A, Held MA, Dardaei L, Friboulet L, et al. Primary Patient-Derived Cancer Cells and Their Potential for Personalized Cancer Patient Care. Cell reports. 2017; 21: 3298-3309.

10. Endo H, Okami J, Okuyama H, Kumagai T, Uchida J, Kondo J, et al. Spheroid culture of primary lung cancer cells with neuregulin 1/HER3 pathway activation. Journal of thoracic oncology: official publication of the International Association for the Study of Lung Cancer. 2013; 8: 131-139.

11. Lee SH, Lee EJ, Min KH, Hur GY, Lee SH, Lee SY, et al. Quercetin Enhances Chemosensitivity to Gemcitabine in Lung Cancer Cells by Inhibiting Heat Shock Protein 70 Expression. Clinical lung cancer. 2015; 16: e235-e243.

12. Zheng $\mathrm{C}$, Sun $\mathrm{YH}$, Ye XL, Chen HQ, Ji HB. Establishment and characterization of primary lung cancer cell lines from Chinese population. Acta pharmacologica Sinica. 2011; 32: 385-392.

13. Zhang Z, Wang H, Ding Q, Xing Y, Xu Z, Lu C, et al. Establishment of patientderived tumor spheroids for non-small cell lung cancer. 2018; 13: e0194016.

14. Jiang Y, Zhao J, Zhang Y, Li K, Li T, Chen X, et al. Establishment of lung cancer patient-derived xenograft models and primary cell lines for lung cancer study. Journal of translational medicine. 2018; 16: 138

15. Mosmann T. Rapid colorimetric assay for cellular growth and survival: application to proliferation and cytotoxicity assays. Journal of immunological methods. 1983; 65: 55-63.

16. Kapalczynska M, Kolenda T, Przybyla W, Zajaczkowska M, Teresiak A, Filas $\mathrm{V}$, et al. 2D and 3D cell cultures - a comparison of different types of cancer cell cultures. Archives of medical science: AMS. 2018; 14: 910-919.

17. Baker FL, Spitzer G, Ajani JA, Brock WA, Lukeman J, Pathak S, et al. Drug and radiation sensitivity measurements of successful primary monolayer culturing of human tumor cells using cell-adhesive matrix and supplemented 
medium. Cancer research. 1986; 46: 1263-1274.

18. Green LM, Reade JL, Ware CF. Rapid colorimetric assay for cell viability: application to the quantitation of cytotoxic and growth inhibitory lymphokines. Journal of immunological methods. 1984; 70: 257-268.

19. Gao B, Huang C, Kernstine K, Pelekanou V, Kluger Y, Jiang T, et al. Non-malignant respiratory epithelial cells preferentially proliferate from resected non-small cell lung cancer specimens cultured under conditionally reprogrammed conditions. Oncotarget. 2017; 8: 11114-11126.
20. Siegfried JM. Culture of primary lung tumors using medium conditioned by a lung carcinoma cell line. Journal of cellular biochemistry. 1989; 41: 91-95.

21. Annalaura M, Spyropoulos DD, McFee WE, Newton DA, Baatz JE. Cryopreservation and in vitro culture of primary cell types from lung tissue of a stranded pygmy sperm whale (Kogia breviceps). Comparative biochemistry and physiology Toxicology \& pharmacology: CBP. 2012; 155: 136-142.
Austin J Cancer Clin Res - Volume 5 Issue 1 - 2018

ISSN : 2381-909X | www.austinpublishing group.com Zhang et al. (C) All rights are reserved
Citation: Dai Y, Guo S, Huang S, Zhang J and Zhang Z. Summary of Primary Culture of Human Lung Cancer Cells. Austin J Cancer Clin Res. 2018; 5(1): 1083. 